\title{
A systematic review of methods to assess intake of saturated fat (SF) among healthy European adults and children: a DEDIPAC (Determinants of Diet and Physical Activity) study
}

Fiona Riordan ${ }^{1 *}$ (D), Roisin McGann², Ciara Kingston ${ }^{3}$, Ivan J. Perry ${ }^{1}$, Matthias B. Schulze ${ }^{4}$, Lene Frost Andersen ${ }^{5}$, Anouk Geelen ${ }^{6}$, Pieter van't Veer ${ }^{6}$, Simone J. P. M. Eussen ${ }^{7}$, Martien C. J. M. Van Dongen ${ }^{7}$,

Nicole E. G. Wijckmans-Duysens ${ }^{7}$ and Janas M. Harrington ${ }^{1}$

\begin{abstract}
Background: Dietary fat is an essential macronutrient. However, saturated fact has been associated with negative health outcomes including cardiovascular disease. Shifting consumption from saturated fat to unsaturated fats and limiting the level of saturated fat in the diet has been recommended. Currently, there is no standard method to measure saturated fat intake in etiologic studies. Therefore, it is difficult to obtain a reliable picture of saturated fat intake in Europe. To inform the development of the DEDIPAC (DEterminants of Dlet and Physical Activity) toolbox of methods, we aimed to identify the assessment methods and specific instruments which have been used to assess saturated fat intake among children or adults in pan-European studies.

Methods: Three electronic databases were searched for English language studies of any design which assessed intake of saturated fat. Reference lists were hand-searched. Studies were included if they were conducted in two or more European countries, and involved healthy, free-living children and adults.

Results: The review identified 20 pan-European studies which assessed saturated fat intake. Food Frequency Questionnaires $(n=8)$ and diet records $(n=7)$ were most common, followed by $24-h$ recalls $(n=5)$. Methods differed in portion size estimation and the composition data which was used to calculate nutrient intake. Of the instruments used in more than two European countries, five Food Frequency Questionnaires had been specifically tested for validity to assess saturated fat intake; four among adults (Food4me, PURE, IMMIDIET, Health, Alcohol and Psychosocial factors in Eastern Europe (HAPIEE)) and one among children (used by Piqueras et al.).
\end{abstract}

Conclusions: A standardised approach to portion size estimation and a common source of food composition data are required to measure saturated fat intake across Europe effectively. Only five instruments had been used in more than two European countries and specifically tested for validity to assess saturated fat intake. These instruments may be most appropriate to evaluate intake of saturated fat in future pan-European studies. However, only two instruments had been tested for validity in more than one European country. Future work is needed to assess the validity of the identified instruments across European countries.

Keywords: Saturated fat, Dietary assessment, Europe, DEDIPAC

\footnotetext{
* Correspondence: fiona.riordan@ucc.ie

'School of Public Health, University College Cork, Western Road, Cork, Ireland

Full list of author information is available at the end of the article
}

(c) The Author(s). 2018 Open Access This article is distributed under the terms of the Creative Commons Attribution 4.0 International License (http://creativecommons.org/licenses/by/4.0/), which permits unrestricted use, distribution, and reproduction in any medium, provided you give appropriate credit to the original author(s) and the source, provide a link to the Creative Commons license, and indicate if changes were made. The Creative Commons Public Domain Dedication waiver (http://creativecommons.org/publicdomain/zero/1.0/) applies to the data made available in this article, unless otherwise stated. 


\section{Background}

Dietary fat is an essential macronutrient, providing a source of energy and facilitating the absorption of fatsoluble dietary components such as vitamins [1]. Saturated fatty acids (SFA) have been associated with the development of non-communicable diseases, including cardiovascular disease (CVD) [2-4]. The World Health Organisation (WHO) Global Strategy on Diet and Physical Activity recommends shifting consumption from saturated fat (SF) to unsaturated fats, and limiting the level of SF in the diet [5]. The Food and Agriculture Organisation expert consultation on fat and fatty acids in human nutrition has proposed that SFA be replaced by Monounsaturated Fatty Acids (MUFA) and Polyunsaturated Fatty Acids (PUFA) in the diet to reduce the risk of Coronary Heart Disease [4].

The role of SF in the diet has recently been the subject of debate. Some studies suggest SF increases levels of beneficial high-density lipoprotein (HDL). However, whether this offsets the effect of detrimental low-density lipoprotein (LDL), and consequently the risk of CVD, is unclear [6, 7]. To better understand the role of SF in the development of chronic disease there is a need for dietary assessment methods which can measure SF and its contribution to daily energy intake in a reliable and consistent way. However, a number of factors have made cross-country comparisons of macronutrient intake difficult: differences in the methods used to assess dietary intake, different approaches to portion size estimation, and the type of food composition databases (FCD) used to calculate SF intake.

In recent years there has been growing emphasis on the standardisation of food classification systems, including Food Composition Databases (FCD), between European countries. This has been the focus of a number of European projects [8-15], including The Innovative Dietary Assessment Methods in Epidemiological Studies and Public Health (IDAMES) project, which aims to develop new methods to assess dietary intake in Europe [16]. The European Food Safety Authority (EFSA) has recommended the standardized 24-HDR recall method, EPIC-Soft (now known as GloboDiet) $[17,18]$. However, there are no agreed standards with respect to the assessment of macronutrients, including SF, for monitoring purposes or aetiological studies.

Partly in recognition of the lack of agreed standards and methodologies, the DEDIPAC: "DEterminants of DIet and Physical Activity" project [19], aimed to create a toolbox of dietary assessment methods which may be most appropriate to use in pan-European studies [19, 20]. The purpose of the current systematic literature review is to identify the assessment methods and specific instruments which have been used to measure intake of SF in European children or adults in more than one European country.

\section{Methods}

\section{Data sources and study selection}

This review adheres to the guidelines of the Preferred Reporting Items for Systematic Reviews and Meta-Analyses (PRISMA) Statement. The protocol for the review can be accessed from the PROSPERO (CRD42014014175) [21]. A systematic literature search was conducted for panEuropean studies which assessed the intake of SF. SF are fatty acids where the fatty acid chain have predominantly single bonds. They can be classified as short, medium, long and very long chain, and are mainly provided in the diet by animal dairy fats, along with some oils, palm oil and coconut oil [4]. Three databases, PubMed, EMBASE and Web of Science, were searched by FR and RM. Search terms included terms for fats (e.g. dietary fat/ s, saturated fat/s, dietary fatty acid/s, saturated fatty acid/s, volatile fatty acid/s, non-essential fatty acid/s, trans fatty acid/s, short chain fatty acid/s, trans fat/s, animal fat/s, lipid/s), along with keywords for dietary and caloric intake, and terms for European countries. A full copy of the EMBASE search strategy is included in Additional file 1: Figure S1. All searches were limited to literatures in English published from 1990 through to 15th March 2017.

Titles and abstract screening of the articles was conducted by FR and RM. In the event of any uncertainty regarding inclusion, the full text of an article was sourced and reviewed. If FR and RM disagreed on article inclusion during full text review then they consulted a third author, JMH. To be included, studies had to be published in a peer-reviewed scientific journal, conducted in two or more European countries, as defined by the Council of Europe [22], and report on the intake of macronutrient SF. Therefore, studies were excluded if they only reported on fat as a food product (e.g. fat-based spreads, fats and oils). SF intake had to be measured at the individual level. Therefore studies which assessed SF intake at the household level or through analysis of biological samples were excluded. Studies had to be conducted among free-living, healthy populations. If study participants were hospital-based or belonged to a disease or societal sub-group they were excluded. The review was not limited by study design; studies with baseline intervention data, and case-control studies where intake was measured in population-based controls, were included (Fig. 1).

Reference lists of all included papers were hand-searched for additional publications. The names of European projects listed in the DEDIPAC Inventory of Relevant European Studies, were also used to search the databases. If necessary, study authors were contacted to request a copy of full paper, or the instrument or questionnaire.

\section{Data extraction and quality assessment}

A data extraction form was created and piloted. This form recorded the following information from included 


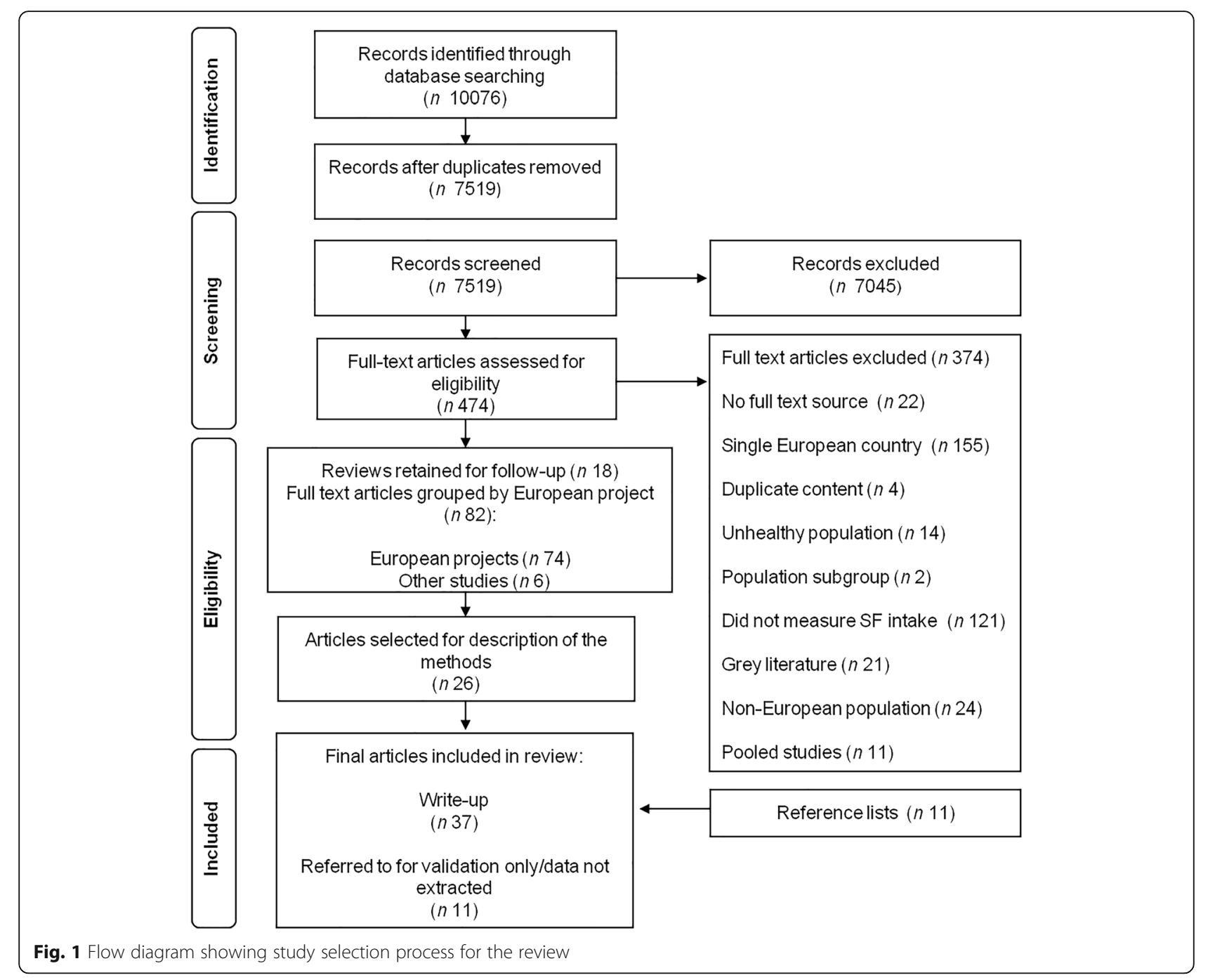

studies: design, number and names of European countries involved, sample size (total and number for each country), age range of the included population, the method used and its description (including frequency categories for Food Frequency Questionnaires (FFQs), details of nutrient intake assessment, details of portion estimation), mode of administration, and details on the validation or reproducibility. Double extraction on each article was carried out by FR and RM. If necessary, further information on the methods was obtained from reference list of the originally included articles.

In line with a previous review of methods to assess fruit and vegetable (F\&V) intake [23], the aim of the current review was to identify instruments. Therefore the quality of each included article was not appraised as part of the current review. Instead, information from the appropriate validation study was extracted by MvD, SE and NW. For an instrument to be considered suitable to assess intake in a pan-European study it had to meet two criteria: 1 . Tested for validity; 2 .Used in more than two countries as part of the same study. These two countries had to represent at least one country from at least three of the Southern, Northern, Eastern, Western European regions as defined by the United Nations [24]. Table 1 shows the results of this assessment.

\section{Results}

\section{Description of the included studies}

In total 10,076 papers were identified. After removing duplicates 7519 remained. Following title and abstract screening and full text review, 82 primary research articles were retained. These articles were organised by the European project to which they belonged. If they did not belong to a project they were grouped as 'Other' $(n=6)$ (see Fig. 1 for a breakdown). 'Study' refers to the larger project, rather than individual articles based on the same project and methodology. Of the 82 articles retained, 26 provided a detailed description of the project or the method in question. These 26 articles were selected for the current review, which equated to 1-3 articles per project. A further 11 articles were sourced from reference lists [14, 25-34]. 


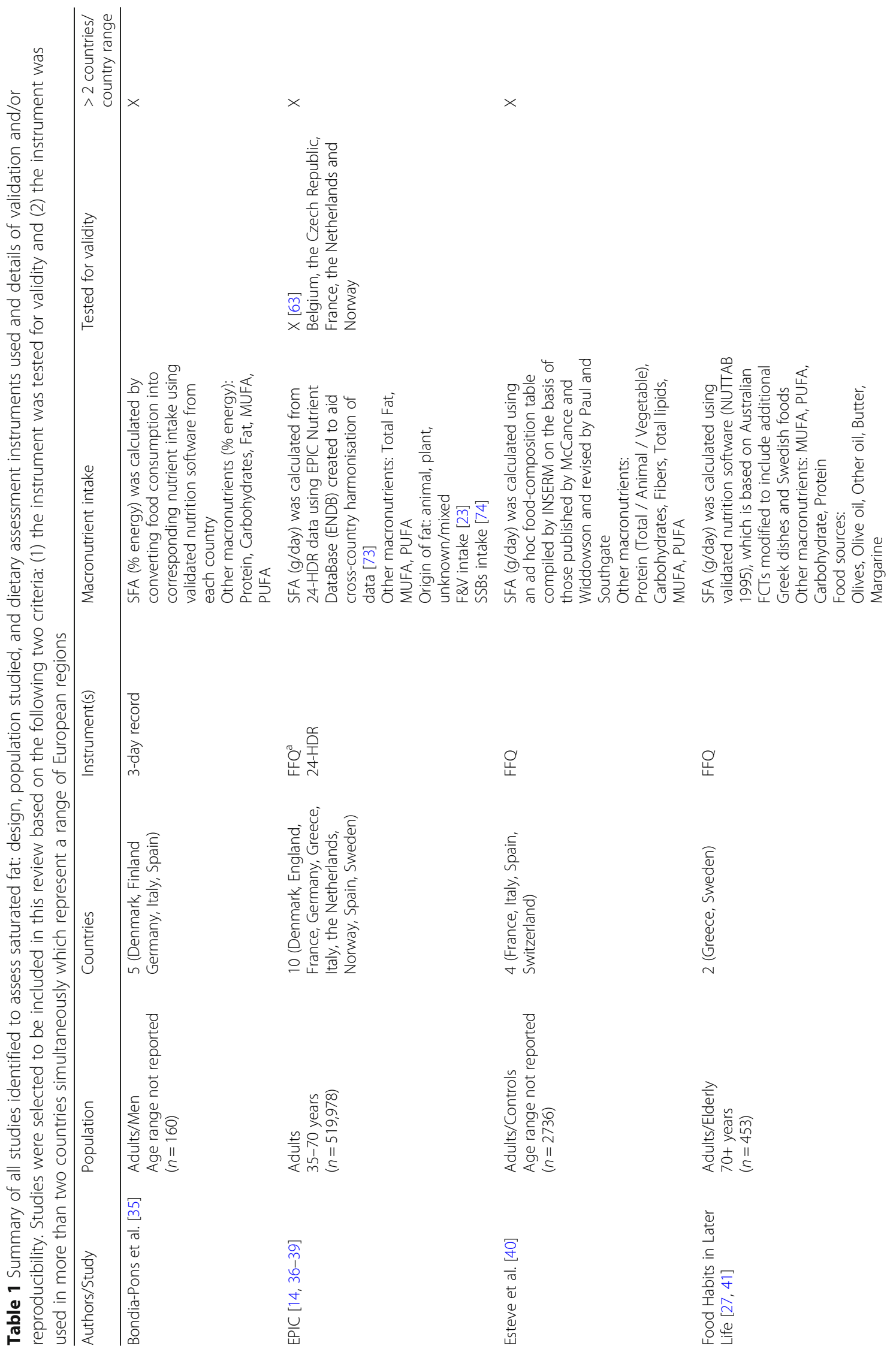




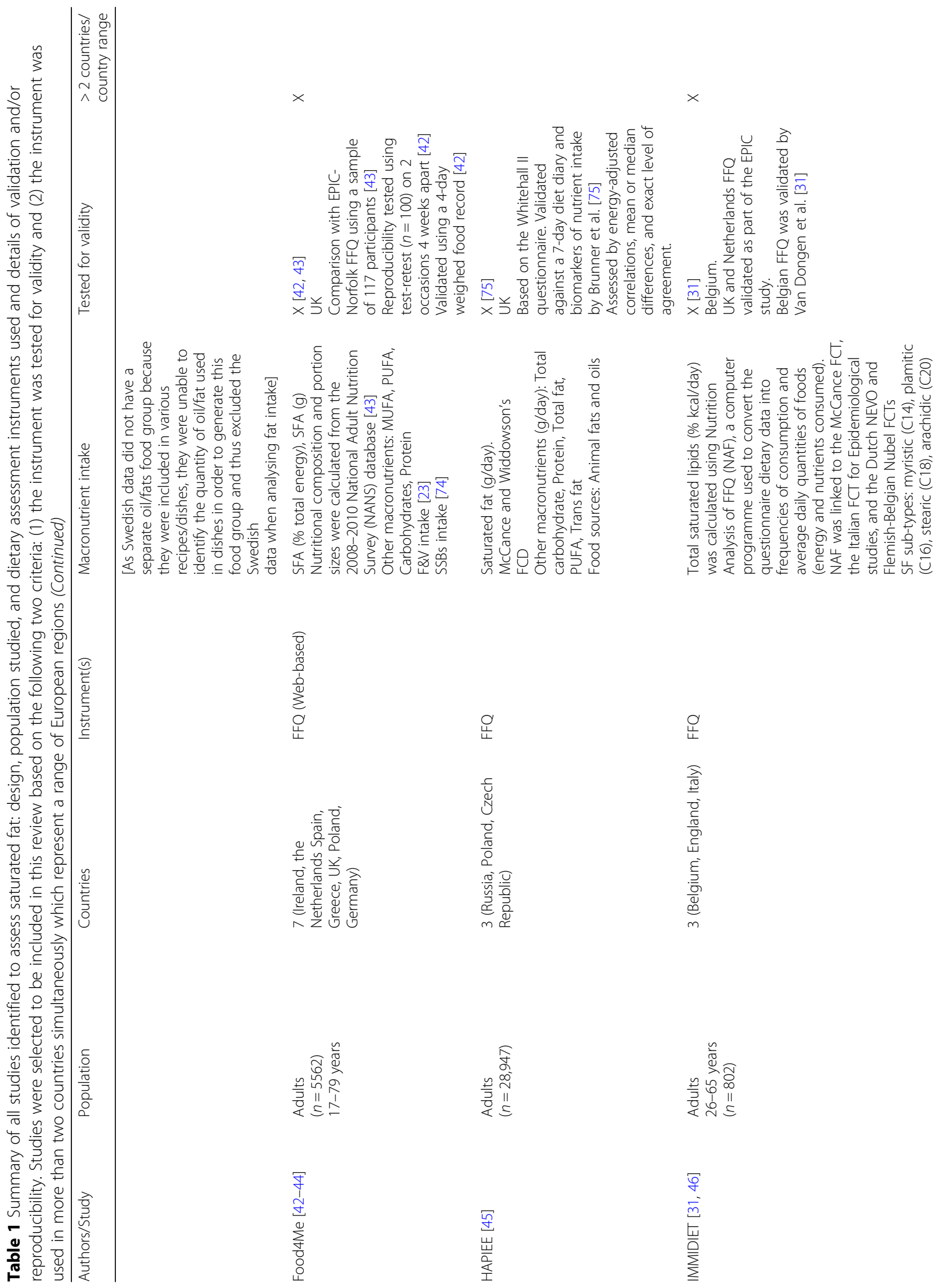




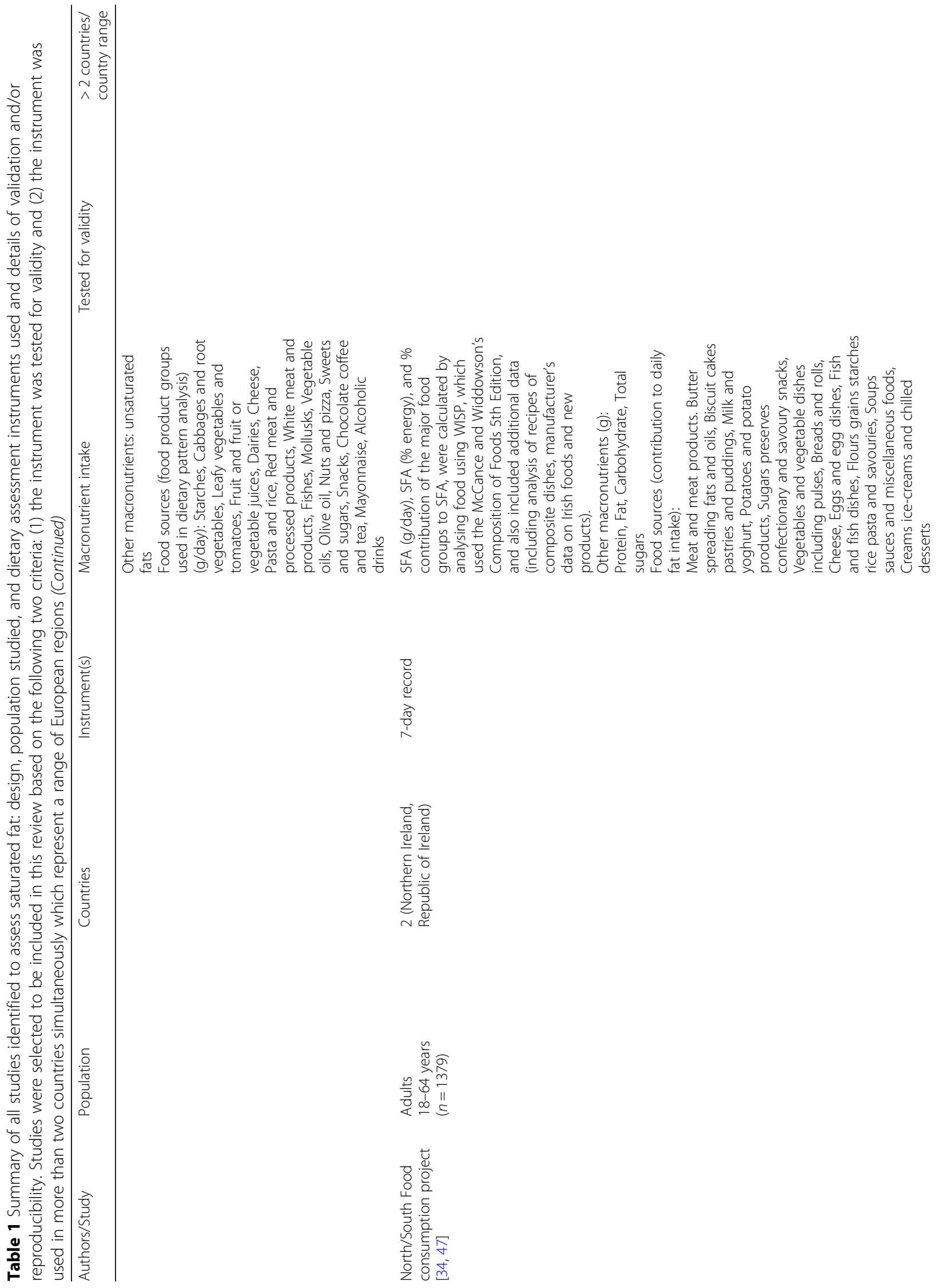




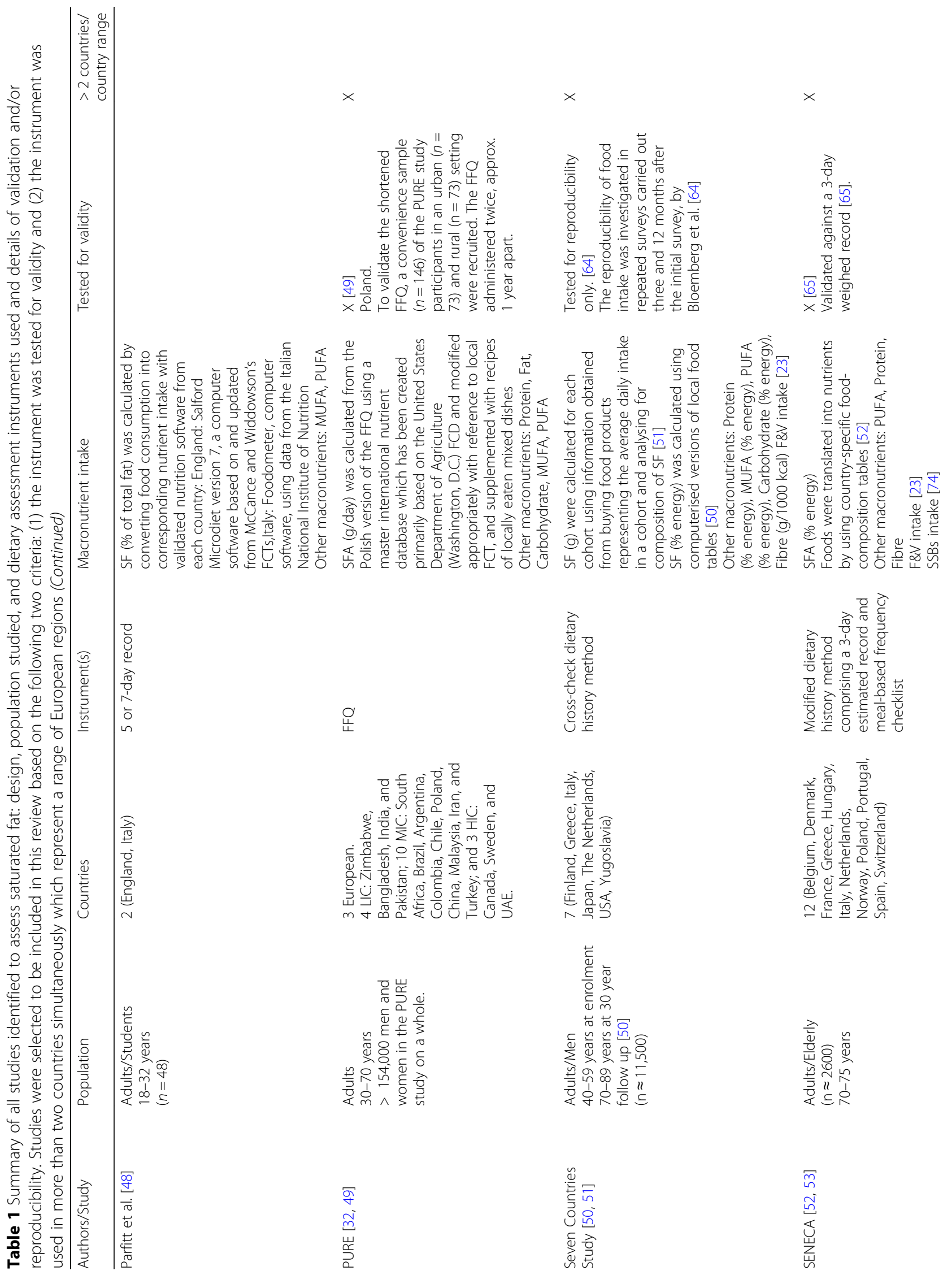




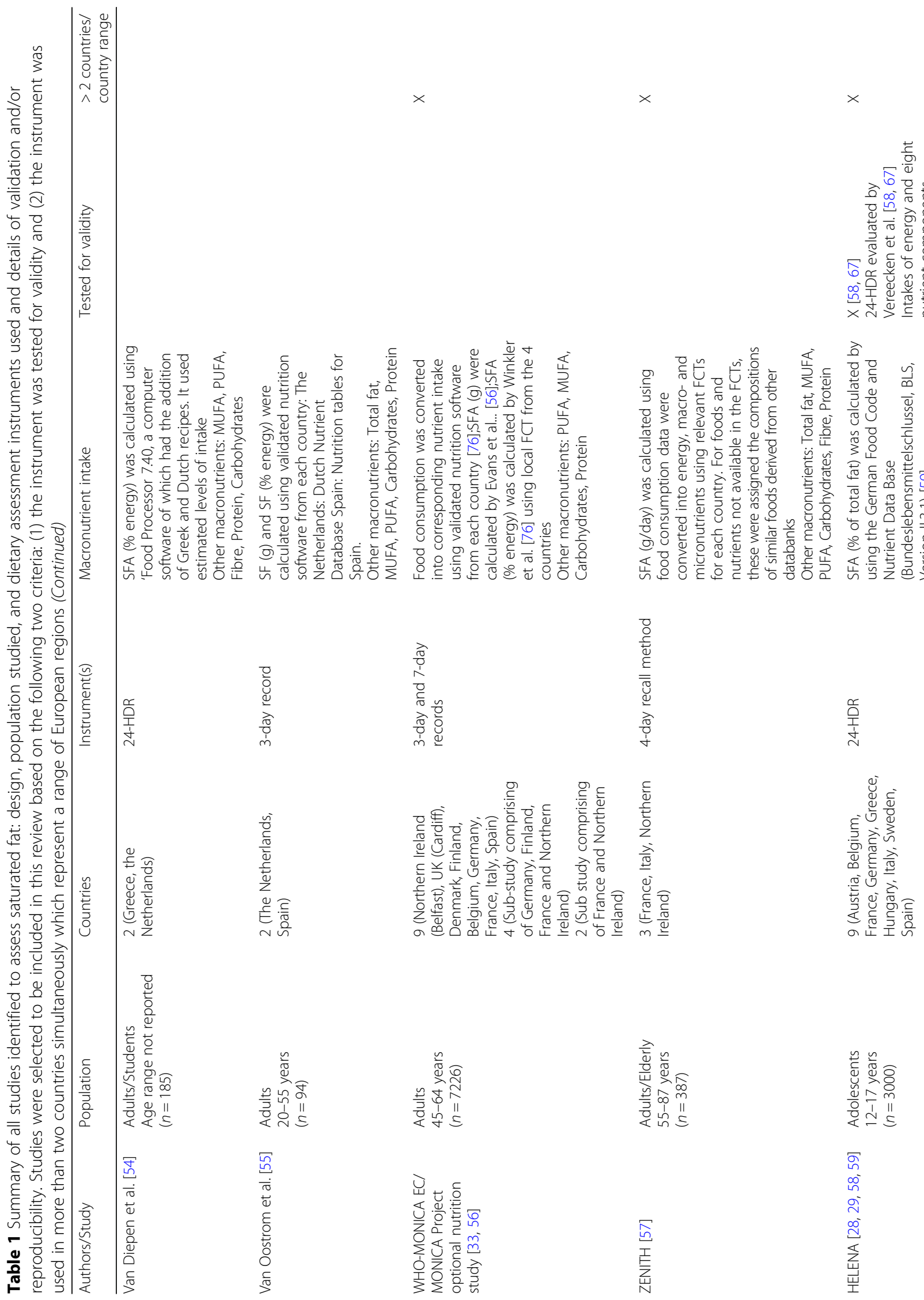




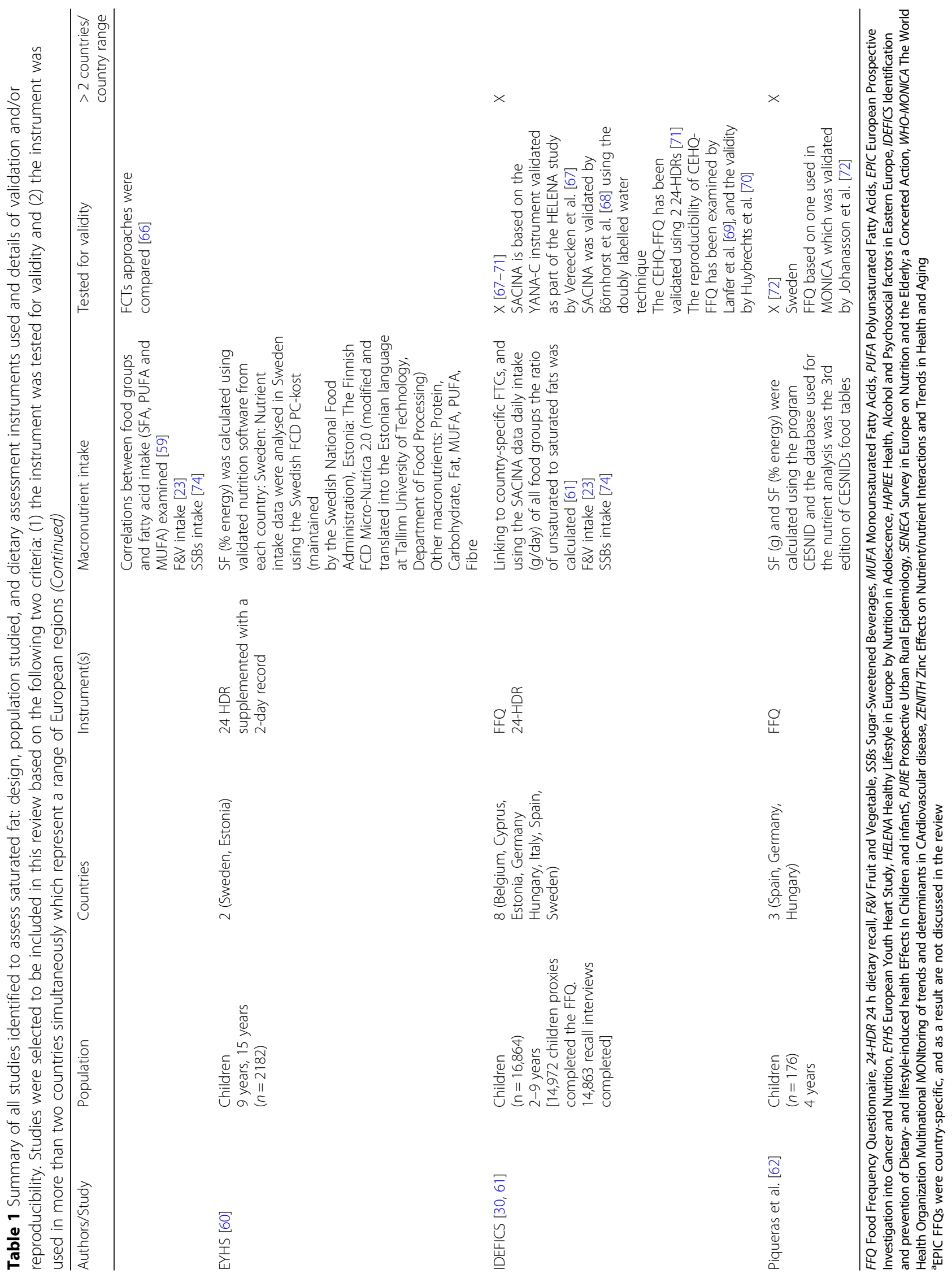


In total, 37 articles [14, 27-62] from 20 studies were included in the review. This number included articles from the original search $(n=26)$, and from reference lists $(n=11)$. Articles in which the instrument was tested for validity were also recorded $(n=11)$ [58, 63-72]. The characteristics of the included studies are described in Table 1. They comprised of large pan-European studies $(n=11)$ and smaller studies conducted in 2-4 countries $(n=6)$. Four studies assessed intake of SF in children [60-62], or adolescents [59], and 13 assessed intake among adults [35, 40, 41, 46-50, 54-57, 73].

\section{Dietary assessment methods \\ Types of methods}

Four approaches to measure SF intake were identified: FFQs, 24 h recalls (24-HDRs), dietary record/diet diaries, or dietary history methods. Most studies used FFQs or 24-HDRs. Several studies used instruments which had been tested for validity: IMMIDIET, European Prospective Investigation into Cancer and Nutrition (EPIC), Prospective Urban Rural Epidemiology (PURE), Healthy Lifestyle in Europe by Nutrition in Adolescence (HELENA), and Identification and prevention of Dietary- and lifestyleinduced health EFfects In Children and infantS (IDEFICS). One study instrument was based on a FFQ which had been used as part of a different study and previously tested for validity [62]. Countries in the EPIC study did not use a common FFQ instrument, therefore only the EPIC-Soft instrument is discussed in this review.

According to the two criteria (Table 1) six study instruments were appropriate to assess intake of SF in future pan-European studies. Two, the EPIC 24-HDR instrument EPIC-Soft, and the cross-check dietary history method used by the Seven Countries Study, had been used to measure intake among adult populations. The HELENA-DIAT instrument had been used among adolescents. The IDEFICS FFQ and 24-HDR, and the FFQ used by Piqueras et al. [62] had been used among children. Table 1 shows the instruments which met the two criteria. Some of these instruments were also used to assess intake of other macronutrients, and/or also met the criteria to assess intake of F\&V [23] or Sugar Sweetened Beverages (SSBs) [74] as determined from two previous reviews. This is also indicated in Table 1.

\section{Validation}

Of the studies which assessed instrument validity and thus fulfilled inclusion criterion 1 (Table 1), only two instruments, EPIC-Soft [63] and the IDEFICS 24-HDR [68], had been tested for validity in more than one country: Belgium, the Czech Republic, France, the Netherlands and Norway in the case of EPIC-Soft [63]; Belgium and Spain in the case of the IDEFICS 24-HDR [68].
Five instruments, all FFQs [31, 42, 43, 49, 72, 75], had been tested specifically for validity to assess SF intake, in Sweden (Piqueras et al. [62]), UK (Food4Me [42, 43]; Health, Alcohol and Psychosocial factors in Eastern Europe (HAPIEE) [45]), and Poland (PURE [49]), and Belgium (IMMIDIET [31]). FFQs [43], repeated 24HDRs $[31,49,72]$ or diet/food records [42, 75] were used as reference methods. Validity was assessed by crude correlations $[31,42,49,72]$, de-attenuated correlation coefficients $[49,72]$, mean or median differences in SF consumption [49, 72, 75], exact level of agreement of SF consumption [31, 49, 75], or Bland and Altman's plots [31, 42, 43, 49].

In four studies, the instrument reproducibility was also tested. Reproducibility was assessed by correlations $[43,49,72]$, mean/median differences, or intraclass correlation coefficient (ICC) [49] between subsequent assessments of FFQs. References for validation studies are provided in Table 1 . Where available, results of the statistical assessments are provided in Table 2.

\section{Instruments tested for validity}

Details of the five instruments, HAPIEE, Food4Me, PURE, IMMIDIET FFQs, and the FFQ used by Piqueras et al., are summarised in Table 3. The HAPIEE FFQ had moderate agreement (0.4-0.6) for SF intake with a 7-day diet diary (7DD) (Spearman's rank correlation: men, $r=0.43$; women, $r=0.56$ ) [75], as did the Food4Me FFQ which was tested for validity using a 4-day weighed food record (Pearson's crude correlation: $r=0.48$ ) [42]. The Food4Me FFQ also had good agreement (>0.6) with the EPIC Norfolk FFQ $(r=0.71)$ [43]. The FFQ used as part of the PURE study had low to moderate agreement with 4 repeated 24HDR (urban: $r=0.42$; rural: $r=0.39$ ) [49] whereas the IMMIDIET FFQ had low agreement $(<0.4)$ with five repeated 24-HDR (men: $r=0.21$; women $=0.34$ ) [31]. Of the instruments used among children, the FFQ used by Piqueras et al. had good to moderate agreement with 10 repeated 24-HDRs (men: $r=0.58$; women: $r=0.54$ ) [72].

\section{Macronutrient assessment}

The identified instruments measured the intake of SFA or SF in grams $[42,50,55,56,62]$, grams/day $[34,40,41,45,49,57]$ or $\%$ contribution to daily energy intake $[34,35,42,46,50,53-55,60,62,76]$, \% of total fat $[48,58]$, and ratio of unsaturated to saturated fats [61]. Of the five instruments which were tested specifically for validity to assess SF, two reported SF as grams/day (HAPIEE [45], PURE [32, 49]), two as grams (Piqueras et al. [62], Food4Me [42-44]), and three as \% energy/day (IMMIDIET [31, 46], Piqueras et al. [62], Food4Me [42-44]).

EPIC used the EPIC Nutrient DataBase (country-specific food composition data standardised across countries) to 


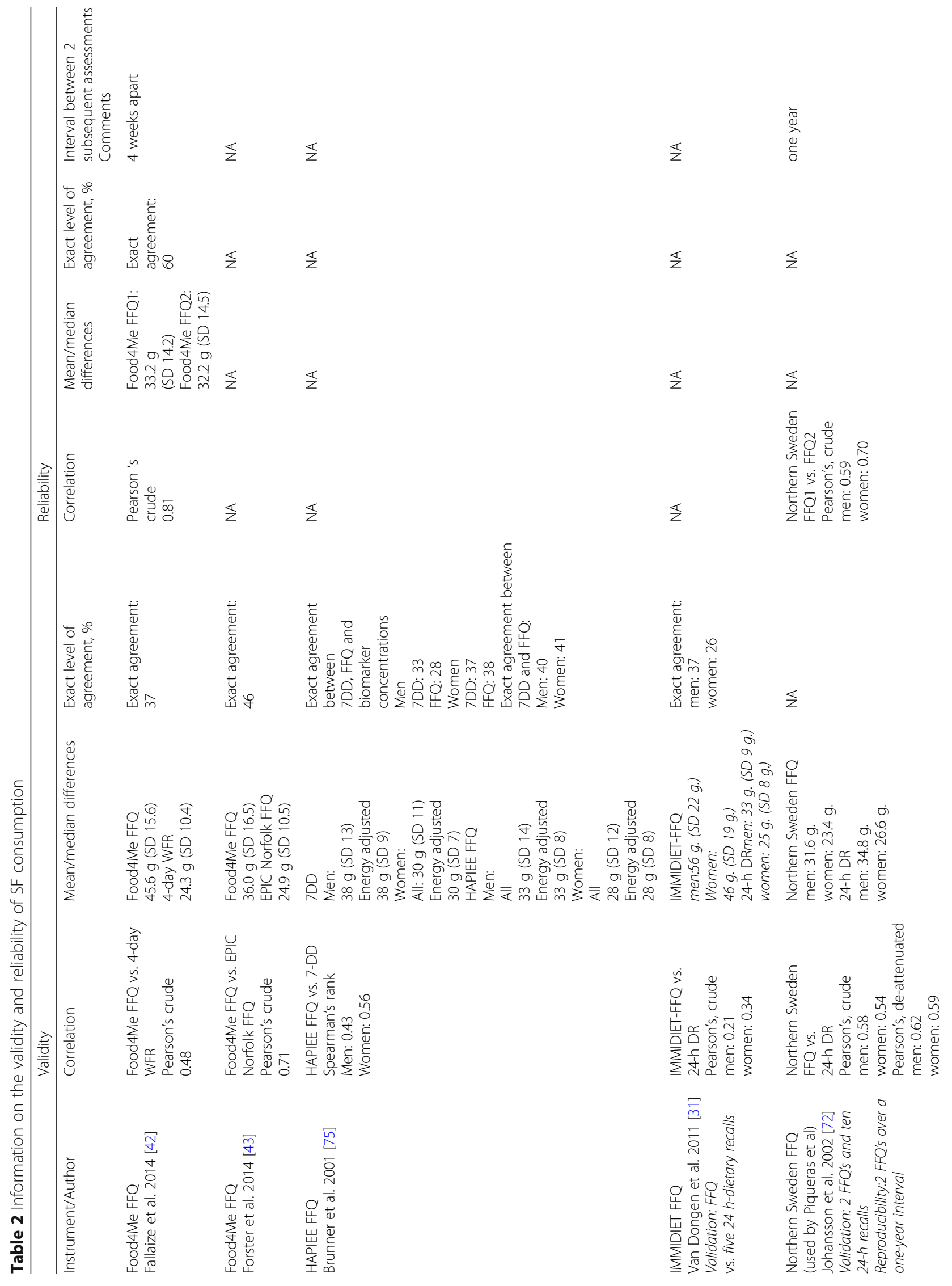




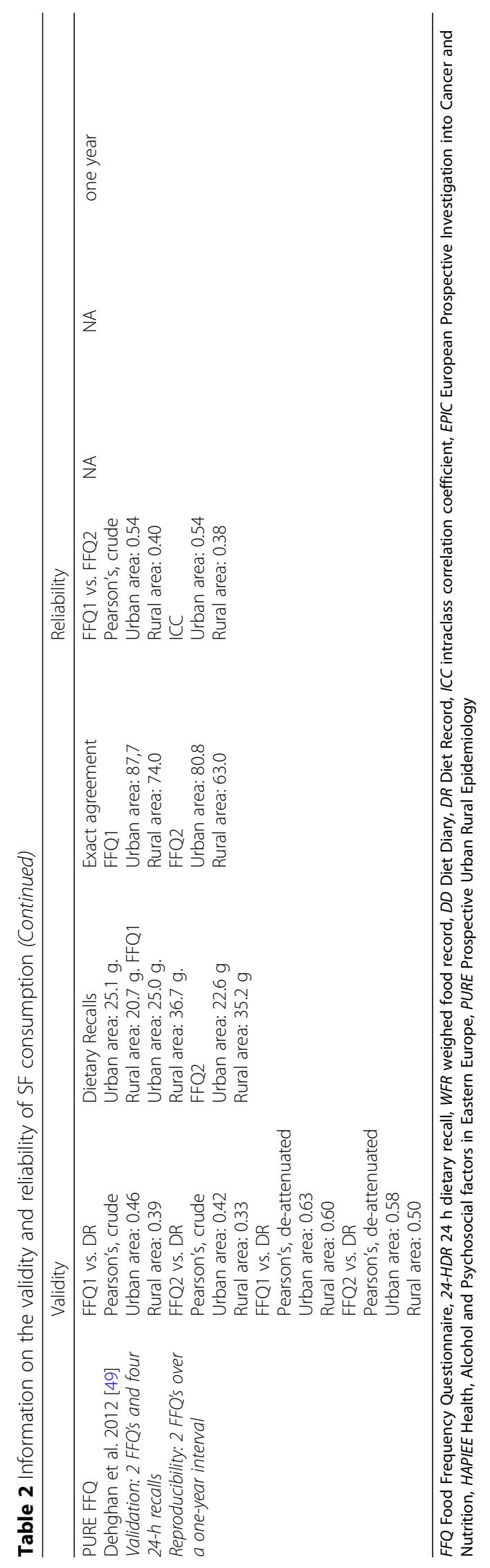


Table 3 Summary of the instruments which were validated $(n=5)$ for assessment of saturated fat

\begin{tabular}{|c|c|c|c|c|c|}
\hline Study/Instrument & Design & Age group & Countries & Mode & Portion estimation \\
\hline \multicolumn{6}{|l|}{ Adults } \\
\hline $\begin{array}{l}\text { HAPIEE [45] FFQ } \\
\text { Validation: 7-day diet diary [75] }\end{array}$ & Cross-sectional & $45-69$ years & 3 (Russia, Poland, Czech Republic) & Self-admin & $x$ \\
\hline $\begin{array}{l}\text { IMMIDIET FFQ [31, 46] } \\
\text { Validation: } 5 \text { 24-HDRs [31] }\end{array}$ & Cross-sectional & $26-65$ years & 3 (Belgium, England, Italy) also in Table 1 & Self-admin. & $x$ \\
\hline $\begin{array}{l}\text { PURE FFQ [32, 49] } \\
\text { Validation: } 2 \text { FFQ's and } 4 \\
\text { 24-HDRs [49] }\end{array}$ & Cross-sectional & 30-70 years & $\begin{array}{l}3 \text { European } \\
4 \text { LIC: Zimbabwe, Bangladesh, India, Pakistan; } \\
10 \text { MIC: South Africa, Brazil, Argentina, } \\
\text { Colombia, Chile, Poland, China, Malaysia, Iran, } \\
\text { Turkey; and } 3 \text { HIC: Canada, Sweden, UAE }\end{array}$ & Self-admin & $x$ \\
\hline $\begin{array}{l}\text { Food4Me FFQ [42-44] } \\
\text { Validation: 4-day weighed food } \\
\text { record [42] }\end{array}$ & $\mathrm{RCT}$ & $17-79$ years & $\begin{array}{l}7 \text { (Ireland, the Netherlands, Spain, Greece, } \\
\text { UK, Poland, Germany) }\end{array}$ & Self-admin & $x$ \\
\hline \multicolumn{6}{|l|}{ Children } \\
\hline $\begin{array}{l}\text { Piqueras et al. FFQ [62] } \\
\text { Validation: } 2 \text { FFQs and } 10 \\
\text { 24-HDR [72] }\end{array}$ & Cross-sectional & 4 years & 3 (Spain, Germany, Hungary) & Self-admin. & $x$ \\
\hline
\end{tabular}

FFQ Food Frequency Questionnaire, 24-HDR $24 \mathrm{~h}$ dietary recall, HAPIEE Health, Alcohol and Psychosocial factors in Eastern Europe, PURE Prospective Urban Rural Epidemiology

calculate intake of SF from dietary data [73]. Mulder et al. [51] (Seven Countries Study), determined SF intake by buying food products which represented the average daily intake in a cohort and analysing these products for composition of SF [51]. The remaining studies used local food composition tables (FCT) from participating countries to calculate intake. In some cases, one FCT was used as the main source of composition data. For example, the German Food Code and Nutrient Data Base (Bundeslebensmittelschlussel) was used by the HELENA study and supplemented with information from the Belgian FCT $[59,77]$.

\section{Food frequency questionnaires (FFQs)}

Characteristics of the FFQ instruments are summarised in Table 4. Where this information was available, the number of FFQs items ranged from to 43 to 322. Most FFQs recorded habitual consumption over the previous year, with the exception of the IDEFICS FFQ [30, 61]. This FFQ assessed intake over a typical week during the previous month. Almost all FFQs were paper-based and self-administered. All were semi-quantitative, and assessed portion size either through specifying a standard portion size on the FFQ for the food item in question [41, 49], or asking participants to consult photos [46], or use household measures [78]. The number of pre-coded frequency categories on the FFQs ranged from 3 to 11 .

\section{Diet records}

Six studies used diet records or diaries (Table 5) to assess SF intake. Portions were estimated using a photo book $[33,34,50,57]$, during the interview (portion description provided by a dietician) [55], using food models [33, 50], or by weighing foods $[34,48,76]$. Most of the identified records were three or seven day records, with one exception, the Zinc Effects on Nutrient/nutrient Interactions and Trends in Health and Aging (ZENITH) study. This study used a four recall day method, over two weekday and two weekend days. All records were self-administered.

\section{Dietary history}

A dietary history approach is an interview-based approach used to record usual intake, asking an individual to recall a typical intake patterns, typically over a longer period (e. g. 6 months) [79]. The Seven Countries Study used a cross-check dietary history method conducted by face-toface interview. The dietary history recorded diet intake in the month preceding the interview. This method had been tested for reproducibility, albeit not specifically to assess SF intake [64]. Usual food consumption pattern was recorded (i.e. foods consumed at breakfast, lunch, dinner and between meals) on a daily basis during week and weekend days. A list of all foods was compiled from this record. Interviewers then recorded what was eaten on a daily, weekly, or monthly basis. A checklist with an extensive number of foods was also used to record the frequencies and amount of foods consumed. Portion size was estimated using different approaches: Finland: photos; The Netherlands: portable scale; Italy: artificial models of different foods in Italy), and also weighed [51].

\section{4 hour dietary recalls (24-HDRs)}

In total, six 24-HDRs were identified, three of which were computerised [61, 73, 80]. Their characteristics are summarised in Table 6. Portion size was estimated for all of identified 24-HDRs, using household measures 


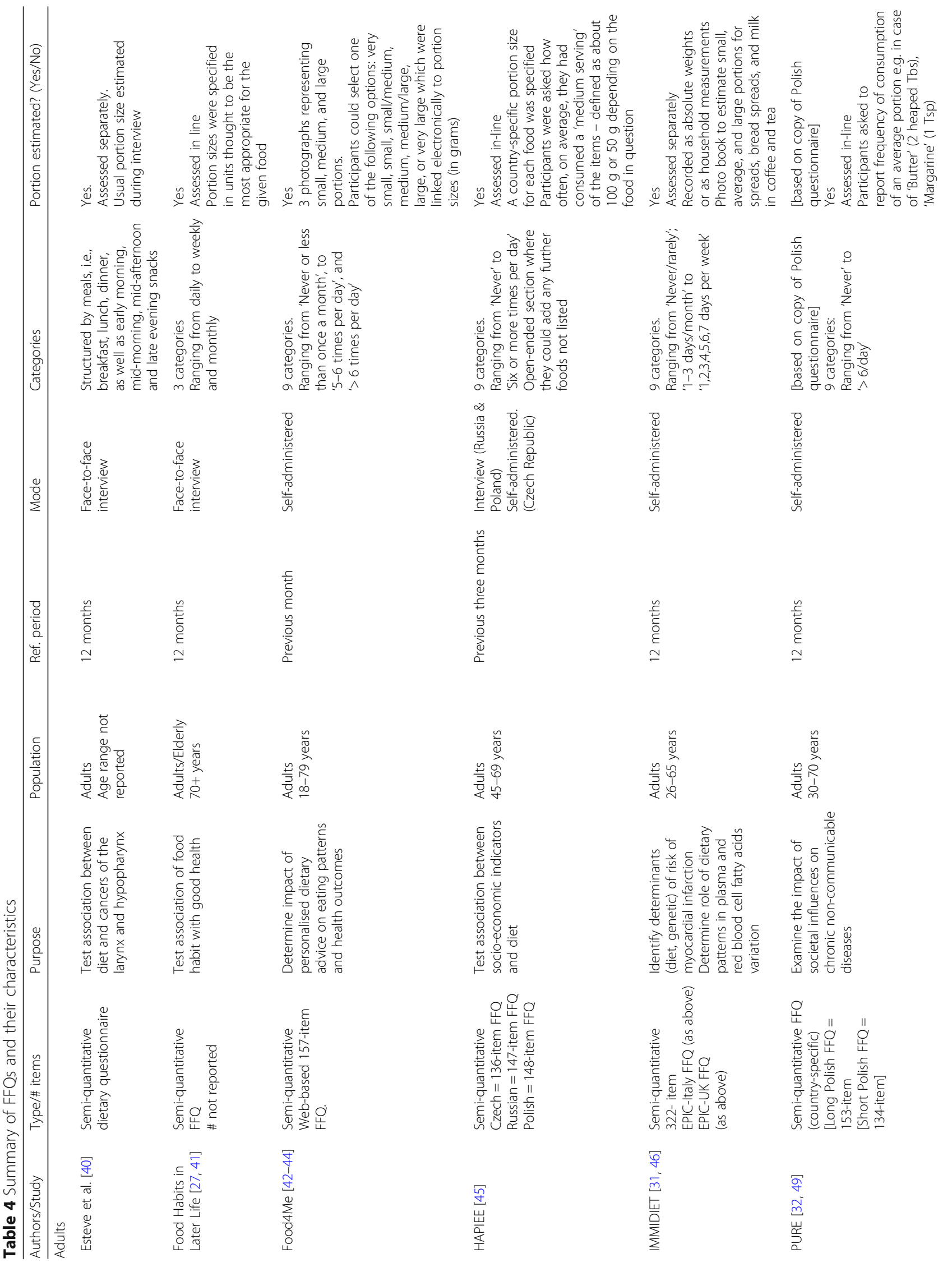




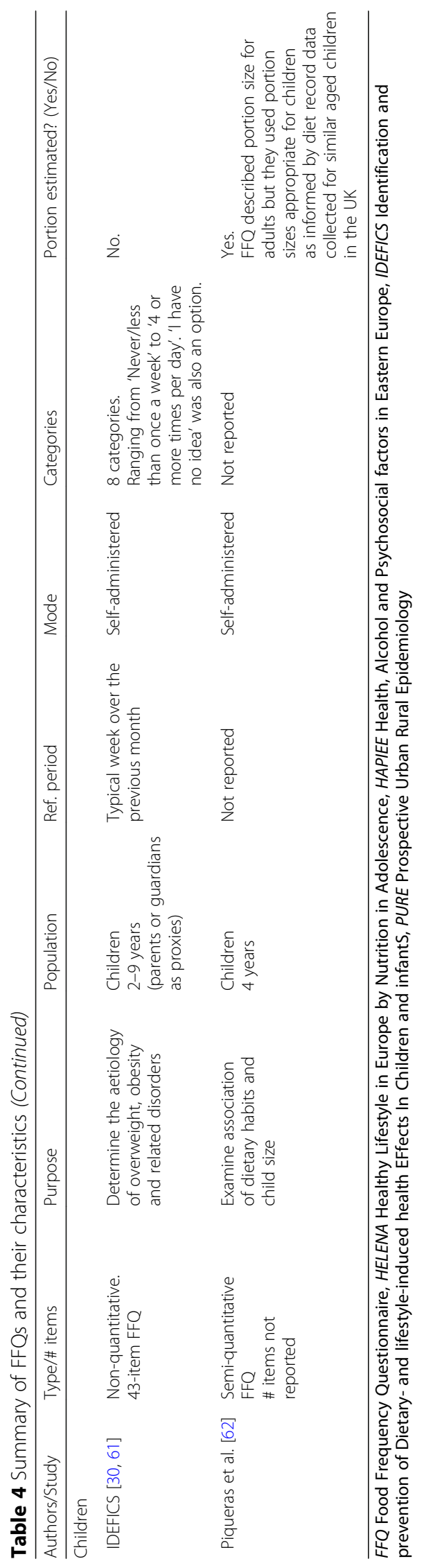




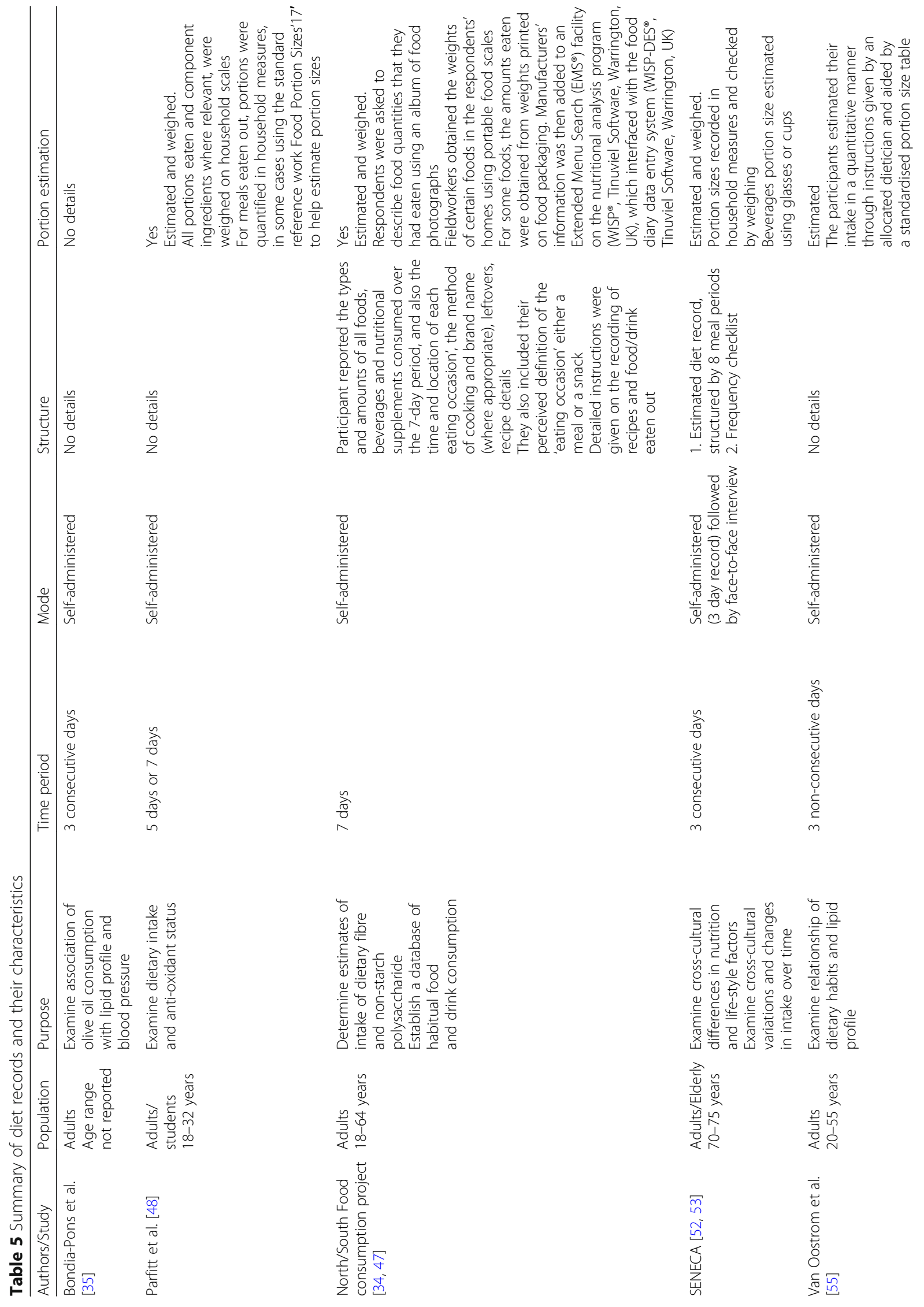




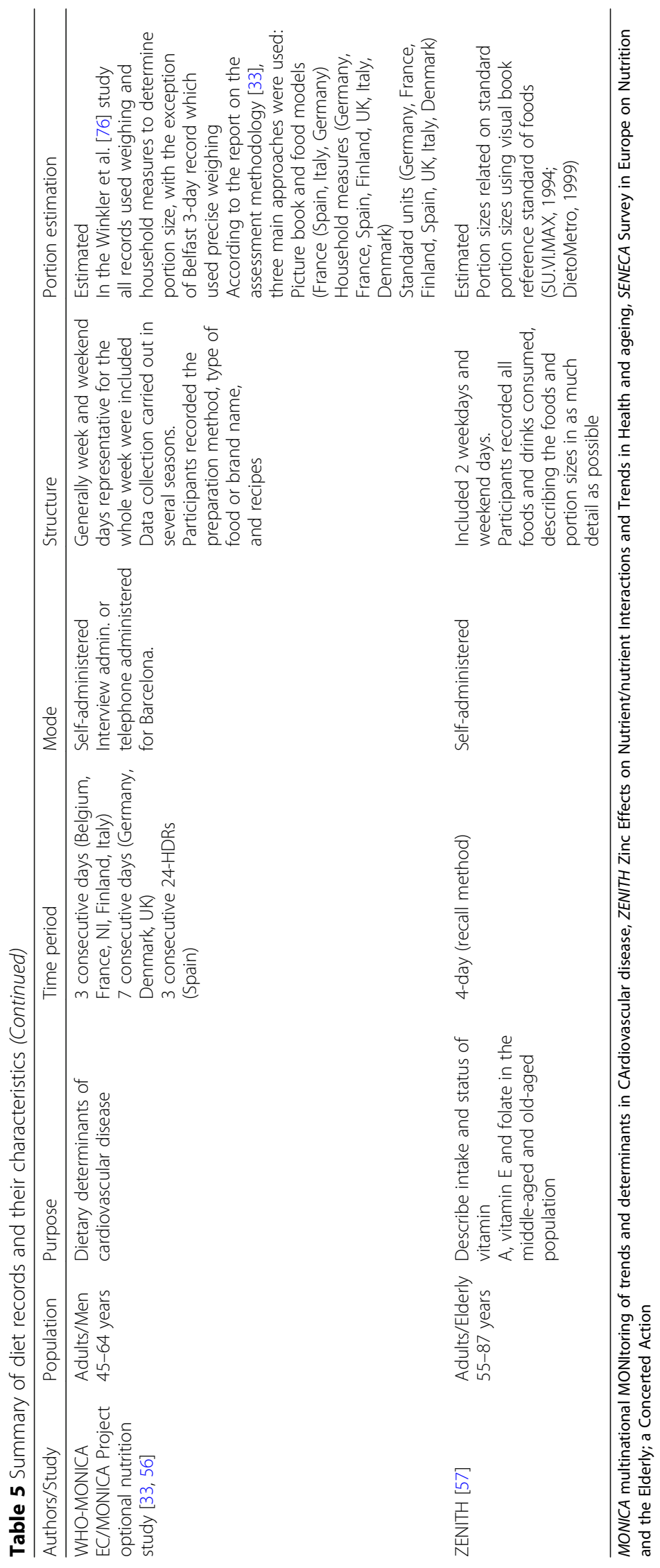




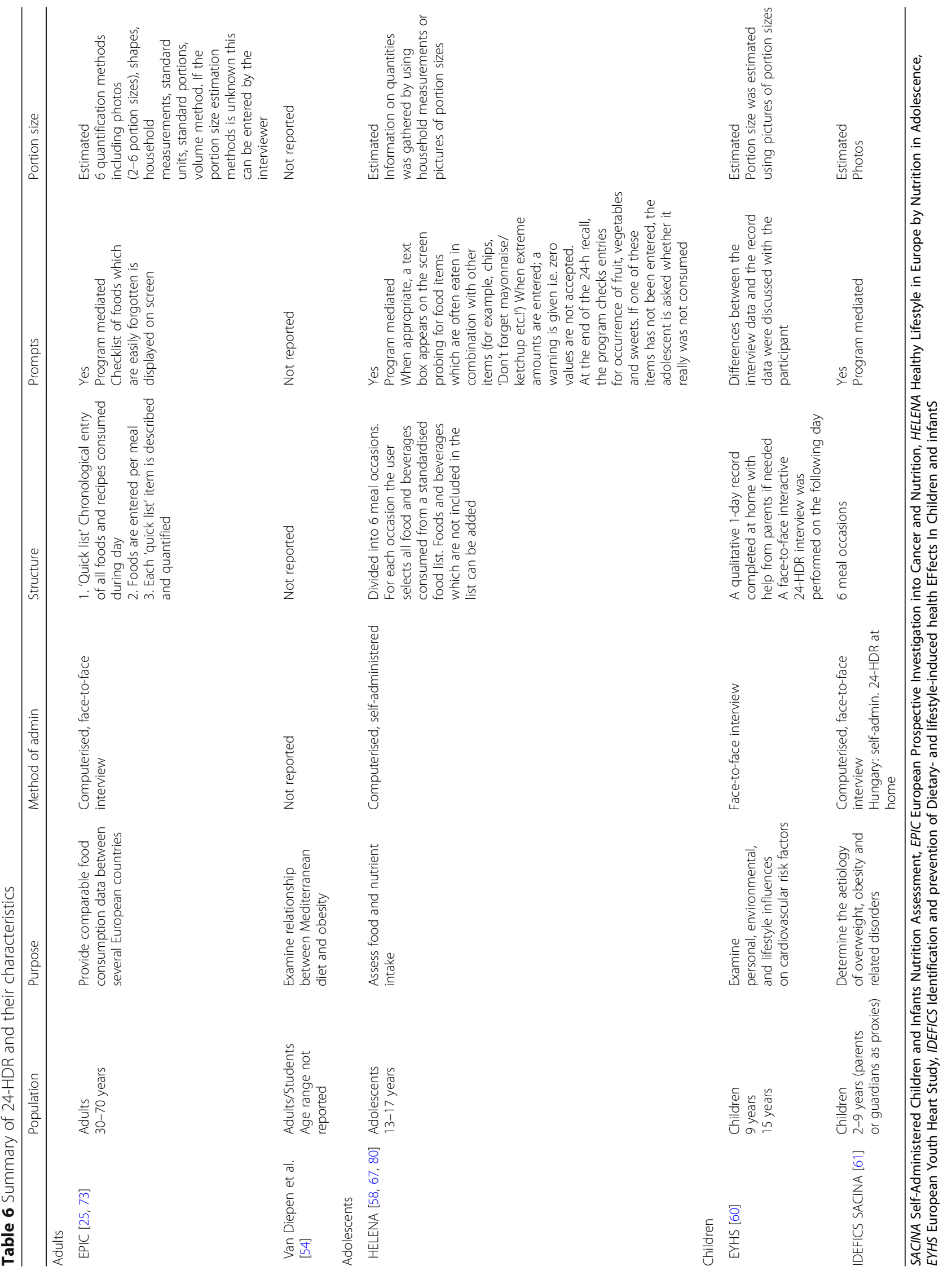


$[80,81]$ or photographs $[60,61,80,81]$. EPIC-Soft, estimated portion sizes using six quantification methods. Two 24-HDRs were tested for validity: HELENA-DIAT [80], which was compared with 1-day food records [58] and tested for reproducibility across administrative modes (self-administration and by interview [67]), and IDEFICS Self-Administered Children and Infants Nutrition Assessment (SACINA) which was tested for validity using the doubly labelled water technique [68].

\section{Discussion}

All four main assessment methods; FFQs, 24-HDRs, diet records/diaries and diet history methods have been used in pan-European studies to measure intake of SF. Of the 20 studies identified, most assessed intake of SF among adults $(n=16)$, and few measured intake among adolescents or children $(n=4)$. While FFQs were most common $(n=8)$, they differed in terms of the approach used to determine portion size and calculate macronutrient intake. Only one identified study, EPIC, used a standardised database as a source of food composition data.

If intake of fat sub-types such as SF and their relationship with disease are to be studied in a standardised way across European countries, it is essential to identify valid instruments. Six study instruments met two criteria (1. the instrument was tested for validity, and; 2 . used in more than two European countries) to assess SF among adults in pan-European studies: the EPIC-Soft 24-HDR, HAPIEE, Food4Me, IMMIDIET, and PURE FFQs, and the SENECA 3-day record. However, only four of these, all FFQs (HAPIEE, IMMIDIET, PURE and Food4Me) had been specifically tested for validity to assess SF intake. Two of these (HAPIEE and Food4Me FFQs) were found to have moderate agreement with diet records. The PURE FFQ had low to moderate agreement with repeated 24-HDRs, and the IMMIDIET FFQ had low agreement with repeated 24-HDRs. Only one identified instrument had been used among adolescents, HELENA-DIAT, but this had not been tested for validity to assess SF intake. Finally, the 24-HDR and FFQ used by the IDEFICS study, and the FFQ used by Piqueras et al. [62] had been used to measure SF intake among children. Of the two, only the FFQ used by Piqueras et al. [62] had been tested for validity to assess SF intake. This instrument had good to moderate agreement with repeated 24-HDRs.

All instruments which had been tested for validity to assess SF intake, had done so using food records (4 and 7 day) $[42,75]$ or $24-$ HDRs $[31,49,72]$ as the reference method. However, using these methods as a reference assumes they are superior in terms of assessing true SF intake. No specific biomarkers for SF exist, therefore, the validity of a 24-HDR to assess true intake cannot be determined. This raises an important question: whether the identified instruments are valid to specifically assess SF intake. Another important consideration is the fact that the level of macronutrient intake may be affected by the source of FCD used for calculations [82]. Ideally pan-European studies would use a common data collection instrument tested for validity, a common approach to portion size estimation, and a standardised source of composition data to calculate intake of SF.

As with previous reviews the results will contribute to the DEDIPAC toolbox of dietary intake assessment methods. The two criteria used in this review, are only an initial approach to identifying suitable instruments. Other factors, including the existing evidence with respect to instrument validity together with instrument feasibility, should be taken into consideration when deciding the appropriateness of an instrument to assess intake of SF in a pan-European population. Only two instruments had been tested for validity in more than one European country. To determine which instruments may be most appropriate, will require further work to test validity across countries. Most identified instruments were also included in two previous reviews on methods to assess intake of F\&V [23] and SSBs [74]. Exceptions were the ZENITH 4-day recall method, PURE FFQ, and the FFQs used by Van Oostrom et al., and Piqueras et al. Overall these two reviews identified a greater number, and variety, of instruments. While this review was limited to pan-European studies, this is not to suggest that other instruments used as part of nonEuropean studies, could not be used to assess intake across Europe.

The review has a number of strengths and limitations. A comprehensive search strategy identified all panEuropean studies measuring intake of SF among children or adults, and the instruments used by these studies. In addition to searching databases, reference lists were hand-searched and study authors were contacted to identify further instruments. A copy of the instrument was sourced in order to accurately describe each instrument. Although the search was comprehensive, it is possible that all relevant articles were not identified. Furthermore, the search was limited to English-language papers. Where a copy of the original instrument or article could not be sourced, the description may be limited, although the results can still be used as a reference. The quality of the identified instruments was not assessed as part of this review. It is important to emphasize that the current review only provides an initial selection of instruments that may be most appropriate to assess SF across European countries. A decision on appropriateness will depend upon instrument validity, which requires further research. Not all SF may be detrimental to health $[83,84]$. In light of this, a final limitation of the review may be the focus on total SF 
intake. Assessing different SFs or subgroups and their relation to health, and reviewing instruments which examine and report on these differences may be an important next step. The majority of the identified instruments evaluated SF as one class. Only one FFQ, used in the IMMIDIET study [31, 46], assessed the intake of SF by sub-types. Lastly, it is important to consider the fact that the identified instruments rely on available food composition data for analysis; the assessment of SF may lag behind changes in food production and composition. FFQs may need to be updated in line with such changes e.g. adding new foods, changing numbers on answer options.

\section{Conclusion}

This review has identified a range of methods to assess intake of SF, FFQs being the most common method used. Key differences exist between the instruments which are currently available to assess SF intake. In order to standardise and harmonise assessment methods between European countries, and increase the accuracy with which intake of SF is measured, it is essential that (1) an agreed method and approach to portion size estimation is used and (2) this is used in conjunction with a standardised source of composition data. This review has indicated five instruments, all FFQs (Food4me, PURE, IMMIDIET, HAPIEE, and FFQ used by Piqueras et al.) which meet both criteria, and were tested for validity to assess SF intake. These instruments may be most suitable to assess intake of SF among healthy populations across Europe. These methods have been used in pan-European populations which encompass a range of European regions, and should be considered by future studies which focus on evaluating SF intake. However, these instruments have only been tested for validity in one country. Future work is needed to test the validity of these instruments across European countries.

\section{Additional file}

Additional file 1: EMBASE search strategy. (PNG 62 kb)

\footnotetext{
Abbreviations

24-HDR: 24 Hour Dietary Recall; CVD: Cardiovascular disease; DD: 7-day diet diary; DD: Diet Diary; DEDIPAC: DEterminants of Dlet and Physical Activity; DR: Dietary Record; EFSA: European Food Safety Authority; EPIC: European Prospective Investigation into Cancer and Nutrition; EYHS: European Youth Heart Study; F\&V: Fruit and vegetable; FCD: Food composition database; FCT: Food composition table; FFQ: Food Frequency Questionnaire; HAPIEE: Health, Alcohol and Psychosocial factors in Eastern Europe; HDL: High-density lipoprotein; HELENA: Healthy Lifestyle in Europe by Nutrition in Adolescence; ICC: Intraclass correlation coefficient; IDAMES: Innovative Dietary Assessment Methods in Epidemiological Studies and Public Health; IDEFICS: Dietary- and lifestyle-induced health EFfects In Children and infantS; LDL: Low-density lipoprotein; MUFA: Monounsaturated Fatty Acids; PRISMA: Preferred Reporting Items for Systematic Reviews and Meta-Analyses; PUFA: Polyunsaturated Fatty Acids; PURE: Prospective Urban
}

Rural Epidemiology; SACINA: Self-Administered Children and Infants Nutrition Assessment; SENECA: Survey in Europe on Nutrition and the Elderly; a Concerted Action; SF: Saturated Fat; SFA: Saturated Fatty Acids; SSBs: Sugarsweetened beverages; WFR: Weighed Food Record; WHO: World Health Organisation; WHO-MONICA: The World Health Organization Multinational MONItoring of trends and determinants in CArdiovascular disease; ZENITH: Zinc Effects on Nutrient/nutrient Interactions and Trends in Health and Aging

\section{Funding}

The preparation of this paper was supported by the DEterminants of Dlet and Physical ACtivity (DEDIPAC) knowledge hub. This work is supported by the Joint Programming Initiative 'Healthy Diet for a Healthy Life'. The funding agencies supporting this work are: The Health Research Board (HRB), Ireland (DEDIPAC/2013/1). The funders had no role in the design of the study and collection, analysis, and interpretation of data and in writing the manuscript.

\section{Authors' contributions}

FR planned and conducted the review, drafted and revised the paper. RM planned and conducted the review, and drafted the paper. CK, IJP, AG, PV, LFA, MS and JMH contributed to the planning, drafted and revised the paper. SE, MVD and NWD conducted the review of validation data, drafted and revised the paper. All authors read and approved the final manuscript.

Ethics approval and consent to participate

Not applicable

\section{Competing interests}

The authors declare that they have no competing interests.

\section{Publisher's Note}

Springer Nature remains neutral with regard to jurisdictional claims in published maps and institutional affiliations.

\section{Author details}

${ }^{1}$ School of Public Health, University College Cork, Western Road, Cork, Ireland. ${ }^{2}$ School of Public Health, Physiotherapy and Population Science, University College Dublin, Dublin, Ireland. ${ }^{3}$ School of Food and Nutritional Sciences, University College Cork, Cork, Ireland. ${ }^{4}$ Department of Molecular Epidemiology, German Institute of Human Nutrition Potsdam-Rehbruecke, Nuthetal, Germany. ${ }^{5}$ Department of Nutrition, Institute of Basic Medical Sciences, University of Oslo, Oslo, Norway. ${ }^{6}$ Division of Human Nutrition, Wageningen University and Research, Wageningen, Netherlands.

${ }^{7}$ Department of Epidemiology of the Faculty of Health, Medicine and Life Sciences, Maastricht University, Maastricht, The Netherlands.

Received: 22 September 2017 Accepted: 2 May 2018

Published online: 08 May 2018

\section{References}

1. EFSA Panel on Dietetic Products, Nutrition and Allergies (NDA). Scientific opinion on dietary reference values for fats, including saturated fatty acids, polyunsaturated fatty acids, monounsaturated fatty acids, trans fatty acids, and cholesterol. EFSA Journal 2010;8(3):1461.

2. Mozaffarian D, Katan MB, Ascherio A, Stampfer MJ, Willett WC. Trans fatty acids and cardiovascular disease. N Engl J Med. 2006;354(15):1601-13.

3. Teegala SM, Willett WC, Mozaffarian D. Consumption and health effects of trans fatty acids: a review. J AOAC Int. 2009;92(5):1250-7.

4. Food and Agricultural Organisation of the United Nations. Fats and fatty acids in human nutrition. Report of an expert consultation. In: FAO food and nutrition paper 91. Geneva: FAO; 2008.

5. Waxman A. WHO global strategy on diet, physical activity and health. Food Nutr Bull. 2004;25(3):292-302.

6. Lamarche B, Couture P. It is time to revisit current dietary recommendations for saturated fat. Appl Physiol Nutr Metab. 2014;39(12):1409-11.

7. Harcombe Z, Baker JS, Cooper SM, Davies B, Sculthorpe N, DiNicolantonio $\mathrm{JJ}$, Grace F. Evidence from randomised controlled trials did not support the introduction of dietary fat guidelines in 1977 and 1983: a systematic review and meta-analysis. Open heart. 2015;2(1):e000196.

8. DAFNE. Data Food Networking. http://ghdx.healthdata.org/record/datafood-networking-databank-dafne. Accessed 6 May 2018. 
9. Castenmiller J, West CE, (eds.). Report of the third annual meeting of the FLAIR Eurofoods-Enfant project, 10- 12 November 1993. Vilamoura, Portugal. Wageningen. FLAIR Eurofoods-Enfant Project (February) 1994.

10. Working Group on Food Data Management and Interchange. In: Schlotke F, Becker W, Ireland J, Moller A, Ovaskainen ML, Monspart J, Unwin I, editors. COST action 99. Research action on food consumption and composition data. Eurofoods recommendations for food composition database management and data interchange; 2000.

11. Norfoods project. A Nordic approach to Food Composition Data. http:// www.foodcomp.dk/norfoods/project_description.html . Accessed 6 May 2018.

12. EFCOSUM group. European food consumption survey method. Final report. The Netherlands: TNO Nutrition and Food Research; 2001.

13. Blanquer M, Garcia-Alvarez A, Ribas-Barba L, Wijnhoven TM, Tabacchi G, Gurinovic M, Serra-Majem L. How to find information on national food and nutrient consumption surveys across Europe: systematic literature review and questionnaires to selected country experts are both good strategies. $\mathrm{Br}$ J Nutr. 2009;101(Suppl 2):S37-50.

14. Riboli E, Kaaks R. The EPIC project: rationale and study design. European prospective investigation into Cancer and nutrition. Int J Epidemiol. 1997; 26(Suppl 1):S6-14.

15. de Boer EJ1, Slimani $N$, van 't Veer $P$, Boeing $H$, Feinberg $M$, Leclercq C, Trolle E, Amiano P, Andersen LF, Freisling H, Geelen A, Harttig U, Huybrechts I, Kaic-Rak A, Lafay L, Lillegaard IT, Ruprich J, de Vries JH, Ocké MC; EFCOVAL Consortium.The European Food Consumption Validation Project: conclusions and recommendations.Eur J Clin Nutr. 2011; 65(Suppl 1):S102-7. https://doi.org/10.1038/ejcn.2011.94.

16. Innovative Dietary Assessment Methods in Epidemilogical Studies and Public Health (IDAMES). Dietary Assessment Methods: State of the Art Report. German Institute of Human Nutrition (DIfE); 2010.

17. European Food Safety Authority. General principles for the collection of national food consumption data in the view of a pan-European dietary survey. EFSA Journal. 2009;7(12):1435.

18. European Food Safety Authority (2014) Guidance on the EU Menu methodology. EFSA Journal. 2014;12(12):3944.

19. Determinants of Diet and Physical Activity Knowledge Hub. https://www. dedipac.eu. Accessed 6 May 2018.

20. Vinceti M, Pellacani G, Malagoli C, Bassissi S, Sieri S, Bonvicini F, Krogh V, Seidenari S. A population-based case-control study of diet and melanoma risk in northern Italy. Public Health Nutr. 2005;8(8):1307-14.

21. Harrington JM, Riordan F, McGann R. What are the assessment methods used to determine dietary intake of saturated fat (SF) in adults (>18 years) and children in European countries, according to pan-European studies involving two or more European countries? In: PROSPERO; 2015.

22. Council of Europe. https://www.coe.int/en/web/portal/home/ . Accessed 6 May 2018.

23. Riordan F, Ryan K, Perry IJ, Schulze MB, Andersen LF, Geelen A, Van't Veer P, Eussen S. A systematic review of methods to assess intake of fruits and vegetables among healthy European adults and children: a DEDIPAC (DEterminants of Dlet and physical activity) study. Public Health Nutrition. 2017;20(3):417-48.

24. Composition of macro geographical (continental) regions, geographical sub-regions, and selected economic and other groupings. https://unstats.un. org/unsd/methodology/m49/. Accessed 6 May 2017.

25. Slimani N, Deharveng G, Charrondiere RU, van Kappel AL, Ocke MC, Welch A, Lagiou A, van Liere M, Agudo A, Pala V, et al. Structure of the standardized computerized 24-h diet recall interview used as reference method in the 22 centers participating in the EPIC project. European prospective investigation into Cancer and nutrition. Comput Methods Prog Biomed. 1999;58(3):251-66.

26. Riboli E, Hunt KJ, Slimani N, Ferrari P, Norat T, Fahey M, Charrondiere UR, Hemon B, Casagrande C, Vignat J, et al. European prospective investigation into Cancer and nutrition (EPIC): study populations and data collection. Public Health Nutr. 2002;5(6B):1113-24.

27. Wahlqvist ML: The IUNS cross-cultural study of "food habits in later life"- an overview of key findings. 1995.

28. Moreno LA, De Henauw S, Gonzalez-Gross M, Kersting M, Molnar D, Gottrand F, Barrios L, Sjostrom M, Manios Y, Gilbert CC, et al. Design and implementation of the healthy lifestyle in Europe by nutrition in adolescence cross-sectional study. Int J Obes (Lond). 2008;32(Suppl 5):S4-11.

29. Moreno LA, Gonzalez-Gross M, Kersting M, Molnar D, de Henauw S, Beghin L, Sjostrom M, Hagstromer M, Manios Y, Gilbert CC, et al. Assessing, understanding and modifying nutritional status, eating habits and physical activity in European adolescents: the HELENA (healthy lifestyle in Europe by nutrition in adolescence) study. Public Health Nutr. 2008;11(3):288-99.

30. Ahrens W, Bammann K, Siani A, Buchecker K, De Henauw S, lacoviello L, Hebestreit A, Krogh V, Lissner L, Marild S, et al. The IDEFICS cohort: design, characteristics and participation in the baseline survey. Int J Obes (Lond). 2011;35(Suppl 1):S3-15.

31. van Dongen MC, Lentjes MA, Wijckmans NE, Dirckx C, Lemaitre D, Achten W, Celis M, Sieri S, Arnout J, Buntinx F, et al. Validation of a food-frequency questionnaire for Flemish and Italian-native subjects in Belgium: the IMMIDIET study. Nutrition. 2011;27(3):302-9.

32. Teo K, Chow CK, Vaz M, Rangarajan S, Yusuf S. The prospective urban rural epidemiology (PURE) study: examining the impact of societal influences on chronic noncommunicable diseases in low-, middle-, and high-income countries. Am Heart J. 2009;158(1):1-7. e1

33. Haveman-Nies A, Bokje E, Ocke M, Kromhout D. MONICA optional study on nutrition: the dietary assessment methodology. The Netherlands: RIVM; 2003.

34. Harrington KE, Robson PJ, Kiely M, Livingstone MB, Lambe J, Gibney MJ. The north/South Ireland food consumption survey: survey design and methodology. Public Health Nutr. 2001;4(5a):1037-42.

35. Bondia-Pons I, Schroder $\mathrm{H}$, Covas Ml, Castellote Al, Kaikkonen J, Poulsen HE, Gaddi AV, Machowetz A, Kiesewetter H, Lopez-Sabater MC. Moderate consumption of olive oil by healthy european men reduces systolic blood pressure in non-mediterranean participants. J Nutr. 2007;137(1):84-7.

36. Allen NE, Appleby PN, Key TJ, Bueno-De-Mesquita HB, Ros MM, Kiemeney LALM, Tjonneland A, Roswall N, Overvad K, Weikert S, et al. Macronutrient intake and risk of urothelial cell carcinoma in the European prospective investigation into cancer and nutrition. Int J Cancer. 2013;132(3):635-44.

37. Linseisen J, Bergstrom E, Gafa L, Gonzalez CA, Thiebaut A, Trichopoulou A, Tumino R, Sanchez CN, Garcia CM, Mattisson I, et al. Consumption of added fats and oils in the European prospective investigation into Cancer and nutrition (EPIC) centres across 10 European countries as assessed by 24 hour dietary recalls. Public Health Nutr. 2002;5(6 B):1227-42.

38. Sieri S, Krogh V, Ferrari P, Berrino F, Pala V, Thiebaut ACM, Tjonneland A, Olsen A, Overvad K, Jakobsen MU, et al. Dietary fat and breast cancer risk in the European prospective investigation into Cancer and nutrition. Am J Clin Nutr. 2008;88(5):1304-12.

39. Meidtner K, Fisher E, Angquist L, Holst C, Vimaleswaran KS, Boer JMA, Halkjaer J, Masala G, Ostergaard JN, Mortensen LM, et al. Variation in genes related to hepatic lipid metabolism and changes in waist circumference and body weight. Genes and Nutrition. 2014;9(2):385.

40. Esteve J, Riboli E, Pequignot G, Terracini B, Merletti F, Crosignani P, Ascunce $N$, Zubiri L, Blanchet F, Raymond L, et al. Diet and cancers of the larynx and hypopharynx: the IARC multi-center study in southwestern Europe. Cancer Causes Control. 1996;7(2):240-52.

41. Purba MB, Kouris-Blazos A, Wattanapenpaiboon N, Lukito W, Rothenberg EM, Steen BC, Wahlqvist ML. Skin wrinkling: can food make a difference? J Am Coll Nutr. 2001;20(1):71-80.

42. Fallaize R, Forster H, Macready AL, Walsh MC, Mathers JC, Brennan L, Gibney ER, Gibney MJ, Lovegrove JA. Online dietary intake estimation: reproducibility and validity of the Food4Me food frequency questionnaire against a 4-day weighed food record. J Med Internet Res. 2014;16(8):e190.

43. Forster H, Fallaize R, Gallagher C, O'Donovan CB, Woolhead C, Walsh MC, Macready AL, Lovegrove JA, Mathers JC, Gibney MJ, et al. Online dietary intake estimation: the Food4Me food frequency questionnaire. J Med Internet Res. 2014;16(6):e150

44. Celis-Morales C, Livingstone KM, Marsaux CF, Macready AL, Fallaize R, O'Donovan CB, Woolhead C, Forster H, Walsh MC, Navas-Carretero S, et al. Effect of personalized nutrition on health-related behaviour change: evidence from the Food4me European randomized controlled trial. Int J Epidemiol. 2016;46(2):578-88.

45. Stefler D, Pajak A, Malyutina S, Kubinova R, Bobak M, Brunner EJ. Comparison of food and nutrient intakes between cohorts of the HAPIEE and Whitehall II studies. Eur J Pub Health. 2016;26(4):628-34.

46. Pounis G, de Lorgeril M, Salen P, Laporte F, Krogh V, Siani A, Arnout J, Cappuccio FP, van Dongen M, Donati MB, et al. Dietary patterns and fatty acids levels of three European populations. Results from the IMMIDIET study. Nutrition Metabolism and Cardiovascular Diseases. 2014;24(8):883-90

47. Joyce T, Wallace AJ, McCarthy SN, Gibney MJ. Intakes of total fat, saturated, monounsaturated and polyunsaturated fatty acids in Irish children, teenagers and adults. Public Health Nutr. 2009;12(2):156-65. 
48. Parfitt VJ, Rubba P, Bolton C, Marotta G, Hartog M, Mancini M. A comparison of antioxidant status and free radical peroxidation of plasma lipoproteins in healthy young persons from Naples and Bristol. Eur Heart J. 1994;15(7):871-6.

49. Dehghan M, Ilow R, Zatonska K, Szuba A, Zhang X, Mente A, Regulska-llow B. Development, reproducibility and validity of the food frequency questionnaire in the Poland arm of the prospective urban and rural epidemiological (PURE) study. J Hum Nutr Diet. 2012;25(3):225-32.

50. Virtanen SM, Feskens EJM, Rasanen L, Fidanza F, Tuomilehto J, Giampaoli S, Nissinen A, Kromhout D. Comparison of diets of diabetic and non-diabetic elderly men in Finland, the Netherlands and Italy. Eur J Clin Nutr. 2000;54(3): 181-6.

51. Mulder I, Jansen MC, Smit HA, Jacobs DR Jr, Menotti A, Nissinen A, Fidanza F, Kromhout D. Role of smoking and diet in the cross-cultural variation in lung-cancer mortality: the seven countries study. Seven countries study research group. Int J Cancer. 2000;88(4):665-71.

52. Jankovic N, Geelen A, Streppel MT, de Groot L, Kiefte-de Jong JC, Orfanos P, Bamia C, Trichopoulou A, Boffetta P, Bobak M, et al. WHO guidelines for a healthy diet and mortality from cardiovascular disease in European and American elderly: the CHANCES project. Am J Clin Nutr. 2015;102(4):745-56.

53. de Groot LC, Hautvast JG, van Staveren WA. Nutrition and health of elderly people in Europe: the EURONUT-SENECA study. Nutr Rev. 1992;50(7):185-94.

54. Van Diepen S, Scholten AM, Korobili C, Kyrli D, Tsigga M, Van Dieijen T, Kotzamanidis C, Grammatikopoulou MG. Greater Mediterranean diet adherence is observed in Dutch compared with Greek university students. Nutrition Metabolism and Cardiovascular Diseases. 2011;21(7):534-40.

55. van Oostrom AJ, Real JT, Carmena R, Ascaso JF, Castro Cabezas M. Daylong triglyceridaemia in healthy Mediterranean and northern European subjects. Neth J Med. 2004;62(8):279-85.

56. Evans AE, Ruidavets JB, McCrum EE, Cambou JP, McClean R, Douste-Blazy P, McMaster D, Bingham A, Patterson CC, Richard JL, et al. Autres pays, autres coeurs? Dietary patterns, risk factors and ischaemic heart disease in Belfast and Toulouse. QJM. 1995:88(7):469-77.

57. Polito A, Intorre F, Andriollo-Sanchez M, Azzini E, Raguzzini A, Meunier N, Ducros V, O'Connor JM, Coudray C, Roussel AM, et al. Estimation of intake and status of vitamin a, vitamin $\mathrm{E}$ and folate in older European adults: the ZENITH. Eur J Clin Nutr. 2005;59(SUPPL. 2):S42-7.

58. Vereecken CA, Covents M, Matthys C, Maes L. Young adolescents' nutrition assessment on computer (YANA-C). Eur J Clin Nutr. 2005;59(5):658-67.

59. Vyncke K, Huybrechts I, Van Winckel M, Cuenca Garcia M, Labayen I, Gottrand F, Widhalm K, Leclercq C, Libuda L, Manios Y, et al. Dietary lipid intake only partially influences variance in serum phospholipid fatty acid composition in adolescents: impact of other dietary factors. Lipids. 2014; 49(9):881-93.

60. Villa I, Yngve A, Poortvliet E, Grjibovski A, Liiv K, Sjostrom M, Harro M. Dietary intake among under-, normal- and overweight 9- and 15-year-old Estonian and Swedish schoolchildren. Public Health Nutr. 2007;10(3):311-22.

61. Tognon G, Moreno LA, Mouratidou T, Veidebaum T, Molnar D, Russo P, Siani A, Akhandaf $Y$, Krogh V, Tornaritis $M$, et al. Adherence to a Mediterraneanlike dietary pattern in children from eight European countries. The IDEFICS study. Int J Obes (Lond). 2014;38(Suppl 2):S108-14.

62. Piqueras MJ, Campoy C, Miranda MT, Decsi T, Koletzko B, Emmett PM. Comparison of childhood size and dietary differences at age 4 years between three European countries. Eur J Clin Nutr. 2014:68(7):786-92.

63. Crispim SP, Geelen A, Souverein OW, Hulshof PJ, Ruprich J, Dofkova M, Huybrechts I, De Keyzer W, Lillegaard IT, Andersen LF, et al. Biomarker-based evaluation of two 24-h recalls for comparing usual fish, fruit and vegetable intakes across European centers in the EFCOVAL study. Eur J Clin Nutr. 2011; 65(Suppl 1):S38-47.

64. Bloemberg BP, Kromhout D, Obermann-De Boer GL, Van Kampen-Donker M. The reproducibility of dietary intake data assessed with the cross-check dietary history method. Am J Epidemiol. 1989;130(5):1047-56.

65. Nes M, van Staveren WA, Zajkas G, Inelmen EM, Moreiras-Varela O. Validity of the dietary history method in elderly subjects. Euronut SENECA investigators. Eur J Clin Nutr. 1991;45(Suppl 3):97-104.

66. Julián-Almárcegui $C$, Bel-Serrat $S$, Kersting M, Vicente-Rodriguez G, Nicolas G, Vyncke K, Vereecken C, De Keyzer W, Beghin L, Sette S, et al. Comparison of different approaches to calculate nutrient intakes based upon 24-h recall data derived from a multicenter study in European adolescents. Eur J Nutr. 2016;55(2):537-45.

67. Vereecken CA, Covents M, Sichert-Hellert W, Alvira JMF, Le Donne C, De Henauw S, De Vriendt T, Phillipp MK, Beghin L, Manios Y, et al.
Development and evaluation of a self-administered computerized 24-h dietary recall method for adolescents in Europe. Int J Obes. 2008;32(SUPPL. 5):S26-34.

68. Bornhorst C, Bel-Serrat S, Pigeot I, Huybrechts I, Ottavaere C, Sioen I, De Henauw S, Mouratidou T, Mesana MI, Westerterp K, et al. Validity of 24-h recalls in (pre-)school aged children: comparison of proxy-reported energy intakes with measured energy expenditure. Clin Nutr. 2014;33(1):79-84.

69. Lanfer A, Hebestreit A, Ahrens W, Krogh V, Sieri S, Lissner L, Eiben G, Siani A, Huybrechts I, Loit HM, et al. Reproducibility of food consumption frequencies derived from the Children's eating habits questionnaire used in the IDEFICS study. Int J Obes (Lond). 2011;35(Suppl 1):S61-8.

70. Huybrechts I, Bornhorst C, Pala V, Moreno LA, Barba G, Lissner L, Fraterman A, Veidebaum T, Hebestreit A, Sieri S, et al. Evaluation of the Children's eating habits questionnaire used in the IDEFICS study by relating urinary calcium and potassium to milk consumption frequencies among European children. Int J Obes (Lond). 2011;35(Suppl 1):S69-78.

71. Bel-Serrat S, Mouratidou T, Pala V, Huybrechts I, Bornhorst C, FernandezAlvira JM, Hadjigeorgiou C, Eiben G, Hebestreit A, Lissner L, et al. Relative validity of the Children's eating habits questionnaire-food frequency section among young European children: the IDEFICS study. Public Health Nutr. 2014;17(2):266-76.

72. Johansson I, Hallmans G, Wikman A, Biessy C, Riboli E, Kaaks R. Validation and calibration of food-frequency questionnaire measurements in the northern Sweden health and disease cohort. Public Health Nutr. 2002;5(3): 487-96.

73. Linseisen J, Welch AA, Ocke M, Amiano P, Agnoli C, Ferrari P, Sonestedt E, Chajes V, Bueno-de-Mesquita HB, Kaaks R, et al. Dietary fat intake in the European prospective investigation into Cancer and nutrition: results from the 24-h dietary recalls. Eur J Clin Nutr. 2009;63:S61-80.

74. Riordan F, Ryan K, Perry IJ, Schulze MB, Andersen LF, Geelen A, Van't Veer P, Eussen S. A systematic review of methods to assess intake of sugarsweetened beverages among healthy European adults and children: a DEDIPAC (DEterminants of Dlet and physical activity) study. Public Health Nutrition. 2017;20(4):578-97.

75. Brunner E, Stallone D, Juneja M, Bingham S, Marmot M. Dietary assessment in Whitehall II: comparison of $7 \mathrm{~d}$ diet diary and food-frequency questionnaire and validity against biomarkers. Br J Nutr. 2001;86(3):405-14.

76. Winkler G, Doring A, Keil U, Pietinen P, Arveiler D, Cambou JP, Nuttens C, Richard JL, Evans A, McClean R, et al. Comparison of dietary intakes in four selected European populations. Clin Investig. 1992;70(10):889-95.

77. Maes L, Cook TL, Ottovaere C, Matthijs C, Moreno LA, Kersting M, Papadaki A, Manios Y, Dietrich S, Hallstrom L, et al. Pilot evaluation of the HELENA (healthy lifestyle in Europe by nutrition in adolescence) food-O-meter, a computer-tailored nutrition advice for adolescents: a study in six European cities. Public Health Nutr. 2011;14(7):1292-302.

78. Thanopoulou A, Karamanos B, Angelico F, Assaad-Khalil S, Barbato A, Del Ben M, Djordjevic P, Dimitrijevic-Sreckovic V, Gallotti C, Katsilambros N, et al. Nutritional habits of subjects with type 2 diabetes mellitus in the Mediterranean Basin: comparison with the non-diabetic population and the dietary recommendations. Multi-Centre study of the Mediterranean Group for the Study of diabetes (MGSD). Diabetologia. 2004;47(3):367-76.

79. Moran Fagundez LJ, Rivera Torres A, Gonzalez Sanchez ME, de Torres Aured ML, Perez Rodrigo C, Irles Rocamora JA. Diet history: method and applications. Nutr Hosp. 2015;31(Suppl 3):57-61.

80. Vyncke KE, Libuda L, De Vriendt T, Moreno LA, Van Winckel M, Manios Y, Gottrand F, Molnar D, Vanaelst B, Sjostrom M, et al. Dietary fatty acid intake, its food sources and determinants in European adolescents: the HELENA (healthy lifestyle in Europe by nutrition in adolescence) study. Br J Nutr. 2012;108(12):2261-73.

81. Pomerleau J, McKee M, Robertson A, Kadziauskiene K, Abaravicius A, Vaask S, Pudule I, Grinberga D. Macronutrient and food intake in the Baltic republics. Eur J Clin Nutr. 2001;55(3):200-7.

82. Vaask S, Pomerleau J, Pudule I, Grinberga D, Abaravicius A, Robertson A, McKee M. Comparison of the micro-nutrica nutritional analysis program and the Russian food composition database using data from the Baltic nutrition surveys. Eur J Clin Nutr. 2004;58(4):573-9.

83. Chowdhury R, Warnakula S, Kunutsor $\mathrm{S}$, et al. Association of dietary, circulating, and supplement fatty acids with coronary risk: a systematic review and meta-analysis. Ann Intern Med. 2014;160(6):398-406.

84. Mozaffarian D. The great fat debate: taking the focus off of saturated fat. J Am Diet Assoc. 2011;111(5):665-6. 